\title{
Midday Fogging of Scleral Contact Lenses: Current Perspectives
}

\section{Jennifer Swingle Fogt (iD}

The Ohio State University College of Optometry, Columbus, OH, USA
Correspondence: Jennifer Swingle Fogt The Ohio State University College of Optometry, 338 West I0th Avenue, Columbus, $\mathrm{OH}, 43210-1280$, USA

Tel +l 6/4-292-0882

Email Fogt.78@osu.edu

\begin{abstract}
Midday fogging is a common problem in scleral lens wear, as particles accumulate in the tear reservoir between the posterior surface of the lens and the front of the ocular surface during wear. As particulate waste collects, symptoms of blurred vision and discomfort arise, typically leading patients to remove their lenses for cleaning, refilling with fresh solution, and reinsertion into the eye. The appearance of the particulate can vary, likely due to different causes for midday fogging. Studies which attempted to identify the particulate have given some insight into some of the causes, but larger studies are needed to identify this debris. Research on lens solutions used for filling the lens reservoir and of various aspects of scleral lens fits have also begun to culminate in the concept that midday fogging may ultimately be related to inflammation. Since scleral lens wearers can have varied and multiple sources of inflammation, strategies in minimizing midday fogging can differ between patients.
\end{abstract}

Keywords: midday fogging, scleral lenses, interrupted scleral lens wear, turbidity, post-lens tear reservoir

\section{Introduction}

Scleral lens use has been growing over the past decade. Unlike a smaller diameter corneal gas permeable lens, a scleral lens rests upon the sclera and the reservoir of the lens must be filled with a solution before application to the eye to maintain a post lens tear reservoir between the lens and ocular surface during wear. These large-diameter lenses can provide stable, comfortable vision over an irregular cornea and are an excellent option for patients with ocular surface disease. As the popularity of these lenses grow, practitioners are finding that some scleral lens wearers complain that their vision becomes cloudy over the course of wear, often requiring removal of the lenses to clean and refill with solution before reapplying them to the ocular surface. Studies have confirmed an increase in the turbidity of the post lens tear reservoir after extended periods of scleral lens wear. ${ }^{1}$ This accumulation of particulate in the post-lens reservoir during scleral lens wear, known as midday fogging, can be associated with symptoms of blurred vision and discomfort, and is disruptive to daily life. ${ }^{2}$ Studies have reported that $26 \%$ to $46 \%$ of scleral lens wearers experience midday fogging. ${ }^{3-5}$ Biomicroscopy evaluation of the debris reveals variation in the color and in the appearance of particulate among patients, with sizes of the particulate varying from fine particulate to larger droplets or coalesced, globules. ${ }^{6}$ A patient with midday fogging may have one or more of these types of particulate present in the post-lens reservoir, and the 
variation in the appearance of fogging particulate is likely a result of multiple causes for this condition.

This review describes the limited studies that explore the possible composition of the particulate matter associated with midday fogging, those factors thought to result in midday fogging, and some possible strategies for minimizing midday fogging in scleral lens wear. References for this study were found by searching PubMed for "scleral" and "midday fogging" and further investigation was completed by studying the references to these manuscripts. It should be noted that the literature on midday fogging thus far is minimal, with many manuscripts referencing small studies with minimal subjects and inconclusive outcomes which are likely not reflective of a larger population. Mention of these studies is important since they are cited repeatedly in the literature and among clinicians, but their limitations are discussed in this review so that it is clear that the original source of some statements about midday fogging are based upon small pilot studies and case reports.

\section{Identifying Midday Fogging}

While midday fogging is symptomatically identified as a gradual disruption in vision which requires lens removal, cleaning, and refilling of solution before reinserting, this condition should be verified by visualizing particulate in the post-lens reservoir. Identification of particulate is typically conducted with biomicroscopy, and can also be viewed with anterior optical coherence tomography (OCT) or Scheimpflug images. It should be noted that complaints of progressively worsening blur with scleral lens wear can also be associated with a buildup of debris, such as tear protein, on the front surface of the lens, or more emergently, can be the result of corneal edema. ${ }^{6}$ Evaluation with a biomicroscope during scleral lens wear can differentiate between lens deposits and particulate formation in the post-lens reservoir. It is important to evaluate the ocular surface with and without scleral lenses at all examinations to ensure that variable blur is not associated with active, atopic disease or a disease process that needs treatment.

\section{Particulate Matter}

The particulate which collects in the post-lens reservoir between a scleral lens and the ocular surface is likely comprised of multiple sources. One likely source is the normal regeneration and sloughing of epithelial corneal cells. ${ }^{2}$ Under normal conditions, without lens wear, those epithelial cells that have been sloughed from the ocular surface are removed mechanically by the upper lid during a blink. A scleral lens prevents contact between the eyelid and the cornea, making this removal impossible. While previous studies have shown an inhibition of corneal epithelial cell sloughing in corneal contact lens wear (soft and rigid corneal lenses), no studies have assessed the rate of sloughing when a lens vaults the cornea and requires a fluid reservoir substitution to be utilized. ${ }^{7,8}$ The post-lens reservoir consists of tears and filling solution added to the lens reservoir before the lens is applied to the ocular surface. The filling solution has traditionally been a non-preservative sodium chloride solution. When scleral lenses are initially filled with solution and applied to the eye, the post lens tear film appears clear clinically or when assessed by anterior OCT or Scheimpflug images. Over time, fine particulate can collect ${ }^{1}$ (Figure 1). While it has been shown that there is tear movement during scleral lens wear, ${ }^{9}$ tear exchange is minimal compared to other lens modalities. ${ }^{10}$ As a result, it is likely that sloughed corneal epithelial cells under a scleral lens will accumulate as uninterrupted lens wear continues.

A study of post-lens turbidity with scleral lens wear when filling the lens with preserved saline found that turbidity increased after eight hours of lens wear. ${ }^{11}$ The investigators did not identify the particulate, but they acknowledged that the use of a preservative in the postlens reservoir could have caused an inflammatory response, leading to the secretion of pro-inflammatory molecules that contributed to turbidity. ${ }^{11,12}$

Additionally, corneal epithelial cell sloughing has been shown to increase when the cornea is bathed in solutions with electrolyte compositions different from normal tears, including saline solution which is comprised of sodium and chloride ions. ${ }^{13}$ The composition of the tears includes ions of potassium, calcium, phosphate, magnesium, sodium and bicarbonate. ${ }^{14}$ Bachman et al found that contact of rabbit corneal epithelial cells with solutions which are dissimilar to the tear film can lead to excessive sloughing of corneal epithelial cells. ${ }^{13}$ These investigators reported that bathing the cornea in a solution of sodium and chloride ions, similar to typical saline solution, created a greater rate of sloughing than a solution which was $\mathrm{pH}$ buffered and had an ionic composition similar to that of natural tears. A study by Fullard used a noncontact irrigation chamber to measure the rate of human epithelial cell sloughing and found an increased rate with sodium chloride compared to a solution with a similar ionic 


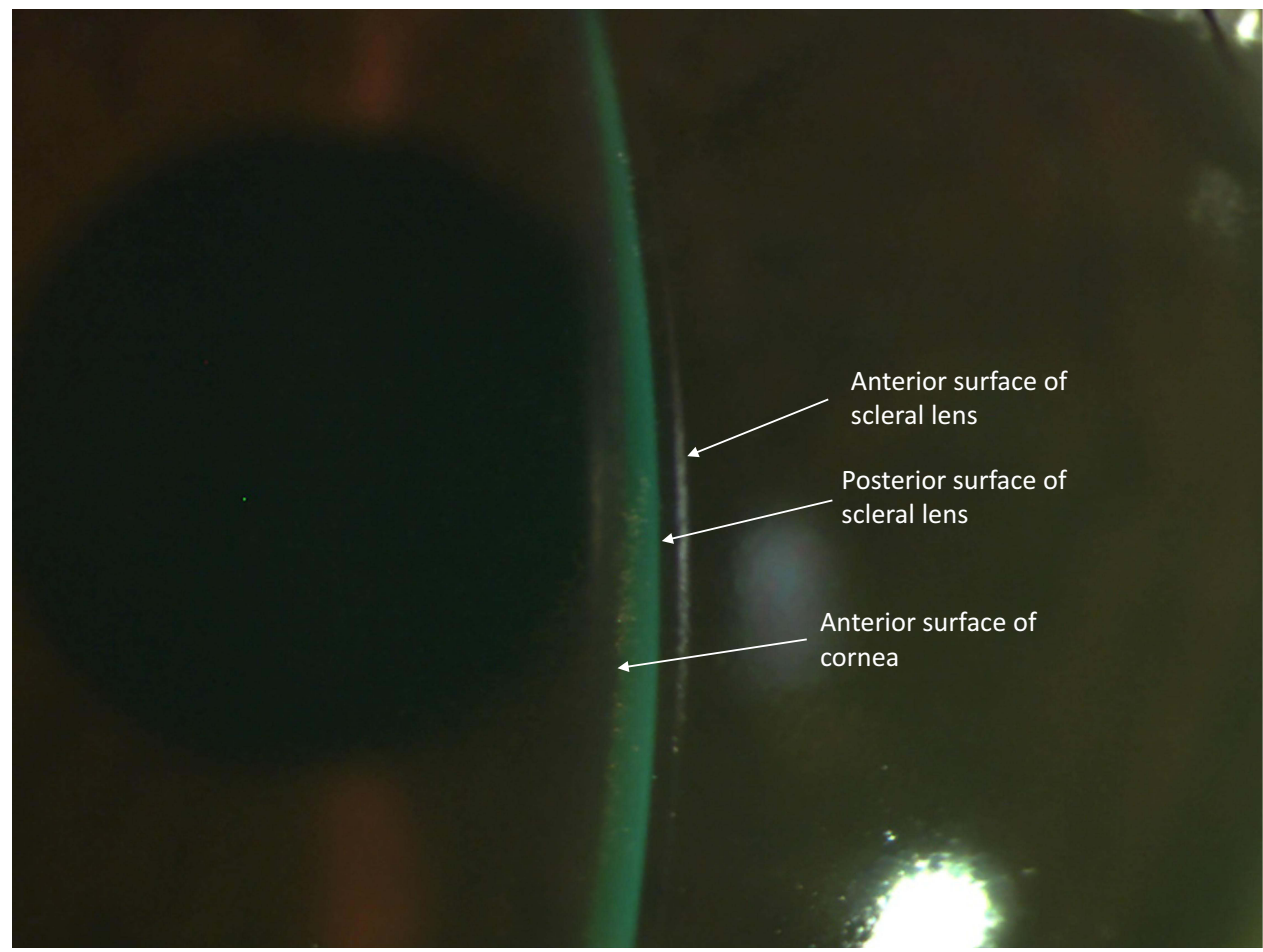

Figure I Midday fogging caused by fine particulate in a patient with keratoconus. In this image, the post-lens tear reservoir, between the scleral lens and the ocular surface, appears green due to the influx of sodium fluorescein after a fluorescein inflow test revealed the presence of tear exchange. This fine particulate normally appears white when examined by biomicroscopy without fluorescein.

composition to tears. The higher squamous cell count found with use of sodium chloride saline indicated that the epithelial cells were not simply the result of removing surface cells that were ready to slough, but that sodium chloride may be causing epithelial cells to slough prematurely $^{15}$ This is of interest, since non-preserved saline, containing only sodium and chloride ions, is commonly used to fill the scleral lens reservoir.

Consequently, a study of scleral lens wearers who experienced midday fogging was conducted to assess safety and efficacy of a scleral lens filling solution with a composition made to mimic $\mathrm{pH}$ and ionic composition of the tears (Nutrifill, Contamac US, Inc, Grand Junction, $\mathrm{CO}){ }^{2}$ Additionally, symptoms in 22 scleral lens wearers were assessed with visual analog scales (VAS) and the Ocular Surface and Disease Index (OSDI) when using the participants' habitual saline as the filling solution and when using the tear ion-mimicking filling solution. Midday fogging graded on a scale of $0-5$ by visual masked-examination of OCT images found a numeric, non-statistically significant decrease with the tear ionmimicking solution. Interestingly, subjective results showed statistically significant improvement in median OSDI scores and VAS scores for blurry/fluctuating vision, dryness, grittiness/foreign body sensation, burning/stinging, and overall pain/discomfort when Nutrifill was used.

A study by Montani quantified the quality of vision in the eyes of 18 habitual scleral lens wearers with keratoconus or post-graft corneas when filling their lenses with unpreserved saline containing potassium, calcium and magnesium electrolytes compared to conventional unpreserved saline. In this study, the visual effects of midday fogging were reduced with the filling solution containing electrolytes which match those in tears. ${ }^{16}$

While studies of corneal epithelial cell sloughing found that increased sloughing occurred in the presence of typical sodium chloride, the exact mechanism by which this occurs is not established. It can be hypothesized that these stressors cause inflammation, which would not only cause an increase in collection of sloughed cells, but could also result in the appearance of inflammatory markers in the post lens tear reservoir. Inflammatory markers, such as matrix metalloproteinase (MMP) 1 and 9, are associated with corneal epithelial cell regeneration. ${ }^{17,18}$ A study by Walker showed that healthy participants had an increase in MMP in the fluid reservoir after wearing scleral lenses for four 8-hour days compared to the MMP in their normal basal tears, concluding that scleral lens wear itself is linked to inflammation. ${ }^{19}$ 
Postnikoff et al studied inflammatory markers in patients who habitually wore scleral lenses. ${ }^{20}$ In this study, 19 habitual scleral lens wearers (39 eyes), with $47 \%$ reporting midday fogging, were evaluated for the presence of leukocytes in the bowl of their lenses and in an eyewash completed upon lens removal. The investigators reported greater mean number of leukocytes present in the participants with midday fogging, although there was no statistical difference in the two groups. It may not be surprising that inflammation is present in the eyes of these habitual scleral lens wearers, particularly since the majority of wearers had keratoconus, which has been linked to the presence of inflammatory markers in recent years. ${ }^{21}$ The extent of midday fogging in individuals with pre-existing inflammatory conditions versus that in individuals who do not have these conditions will need to be considered in future studies.

While the material in midday fogging is commonly described as fine particulate, observation of larger collections of yellow or brown particles (Figure 2) and larger white, wispy clumps (Figures 2 and 3) have been documented and were initially hypothesized to be lipid and mucin, respectively. ${ }^{6}$ These larger coalesced clumps of particulate are also visible by OCT, and have a very

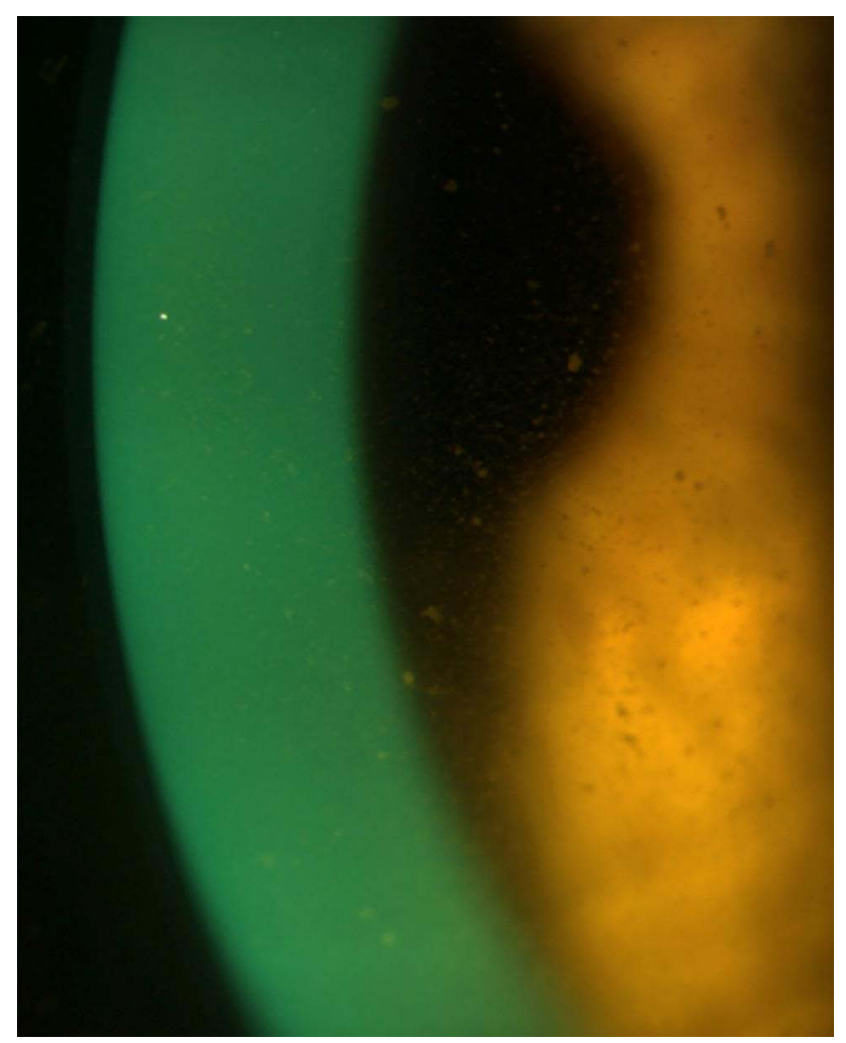

Figure 2 Midday fogging caused by larger, coalesced particulate. The particulate has a yellow or golden appearance, and is likely lipid in this dry eye patient.

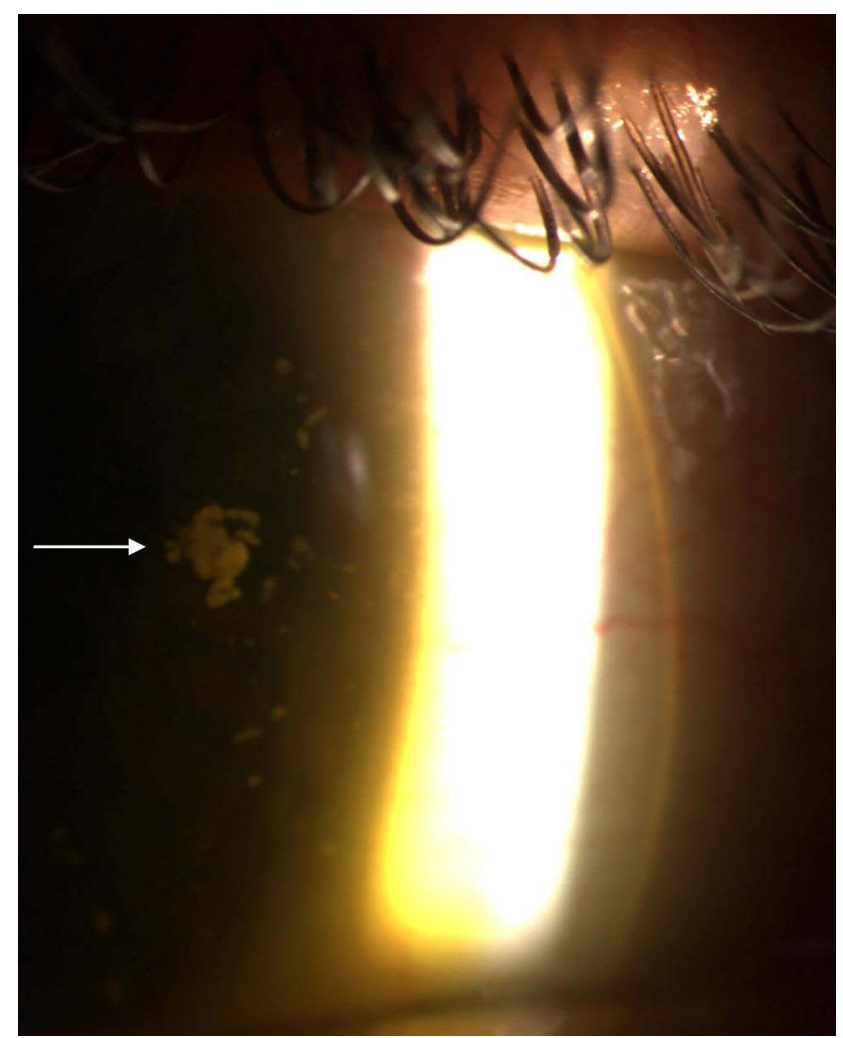

Figure 3 Midday fogging with large, white floating debris. This image captures the presence of a large globule of debris surrounded by smaller globules within the post lens tear reservoir. The arrow indicates a large, coalesced collection of this debris. Initially debris with this appearance was hypothesized to be mucin. No laboratory tests have not yet confirmed the presence of mucin in midday fogging.

different appearance than the fine particulate in midday fogging (Figure 4). A small laboratory analysis of three subjects with turbid post-lens reservoirs and two subjects with non-turbid post-lens reservoirs was conducted by Walker et al in order to attempt to identify the components of midday fogging particulate. ${ }^{22}$ Protein analysis of the post-lens fluid samples was conducted using bicinchoninic acid assay and mass spectrometry and no differences were found when comparing the turbid and non-turbid samples. Lipid detection of the samples using Oil Red O stain, found a large presence of lipid in the turbid samples. In this study, mucin was not identified in the samples from either the fogging or non-fogging wearers. This was of interest, since previous thoughts on midday fogging hypothesized that the larger "white and fluffy" particulate sometimes present in the post lens tear reservoir was mucin produced by the conjunctival goblet cells as a reaction to lens-induced inflammation of the conjunctiva. ${ }^{6}$ Because only three samples with midday fogging were tested in the study, the presence of mucin cannot be ruled out in the general population of scleral 
A

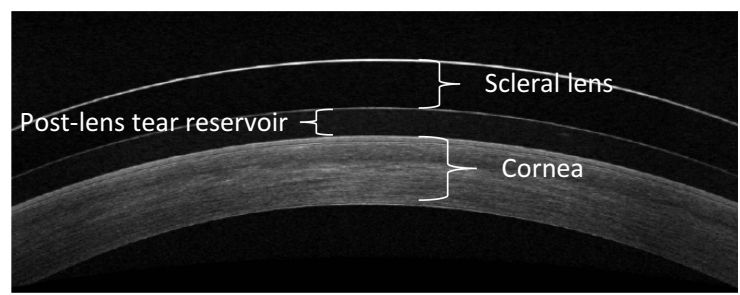

B

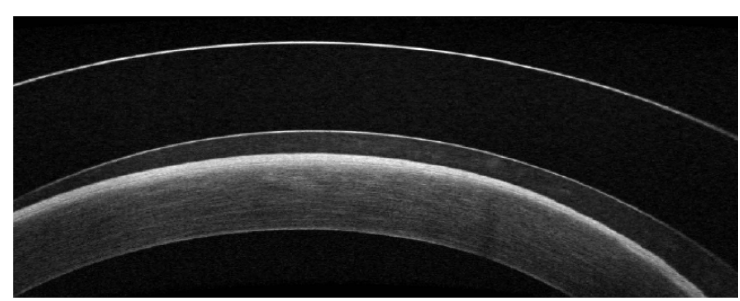

C

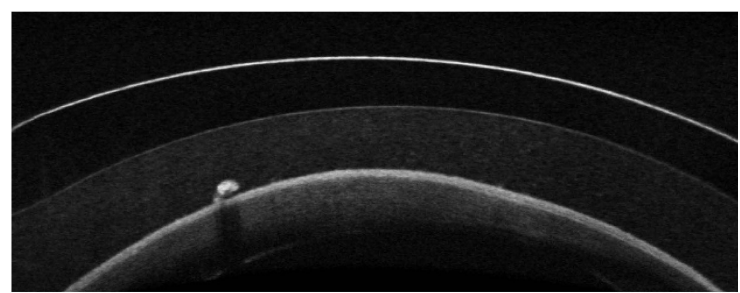

D

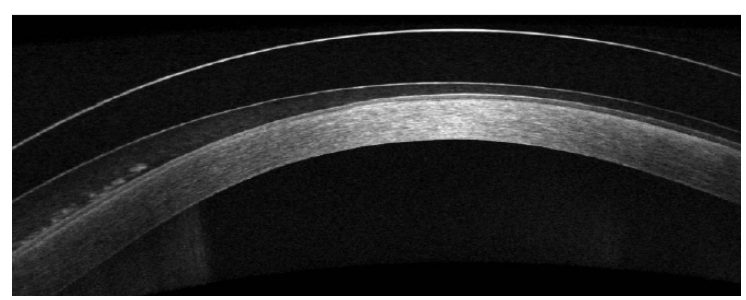

Figure 4 Optical coherence tomography images of the scleral lens, post lens tear reservoir, and cornea. Midday fogging is the accumulation of particulate in the post lens tear reservoir. (A) Minimal particulate is present in the post lens tear reservoir. (B) Dense accumulation of fine particulate is visible in the post lens tear reservoir (C) Fine particulate and one large globule are present in the post lens tear reservoir. (D) Fine particulate and multiple coalesced collections of particulate are visible in the post lens tear reservoir.

lens wearers. The lipid identified in the turbid samples of this study may correspond with the larger yellow or brown oil-like particulate reported in midday fogging, ${ }^{6}$ although Walker's study did not report on appearance of the particulate. The origin of this lipid is unknown. It could be argued that excessive meibum present in the tears is "pumped" under a lens that allows tear exchange. Alternatively, there are complex interactions between inflammation and lipid metabolism, ${ }^{23,24}$ and inflammation has been shown to be present with scleral lens wear. ${ }^{20}$ Because this interaction is not well understood, the presence of lipid in the post lens tear reservoir unrelated to tear exchange cannot be ruled out. Since Walker's small study in which lipid in the post-lens reservoir did not evaluate the edge profile or the presence or absence of tear exchange, the mechanism by which lipid particulate appears in the post lens tear film, is unknown at this time. Further studies are needed with larger sample sizes, evaluation of the meibomian glands, and evaluation for the presence of tear exchange to determine an origin and a possible remedy for the presence of lipid particulate that contributes to midday fogging.

\section{The Role of Scleral Lens Fitting Characteristics in Midday Fogging}

Midday fogging has also been attributed to lens fit characteristics in several studies and case reports, although there is debate about the best practices for fitting lenses to minimize the collection of particulate in the post lens tear film., $31,20,25,26$ These fitting characteristics are described in sub-sections below. The lack of consensus on the fitting parameters that can reduce midday fogging is likely the result of the multi-factorial nature of this problem. In order to minimize the possibility of hypoxia and the resultant inflammation from scleral lens wear, theoretical values for ideal central clearance between the ocular surface and the lens have been identified. ${ }^{27}$ Empirical studies have found less corneal edema than expected from these theoretical predictions. ${ }^{28-30}$ In practice, creating a uniform clearance over a cornea with keratoconus can be challenging, with large areas of vault just adjacent to areas of lesser clearance over the cone, for example. Comparison of clearance between patients and between studies is more straightforward in patients without irregular corneas.

\section{Clearance Over the Ocular Surface}

Some studies have been conducted to determine if the amount of central clearance (ie the thickness of the post lens tear reservoir), created by the vaulted lens over the cornea is related to the presence of midday fogging. A study that evaluated 11 subjects with midday fogging and 12 without fogging found no difference in mean post lens tear thickness by OCT. ${ }^{31}$ Another group evaluated midday fogging particulate utilizing anterior OCT, and found no correlation between the amount of turbidity and clearance of the scleral lenses with 26 subjects. ${ }^{11}$ Conversely, in another study of midday fogging, central clearance was assessed by comparison to lens thickness in 19 habitual scleral lens wearers. The study reported that those with fogging had an average central clearance that was about 50 microns greater than the average clearance 
for those without fogging $(P=0.047) .{ }^{20}$ The study hypothesized that hypoxia caused by increased central clearance could cause the inflammation that in turn causes midday fogging. The investigators did not assess haptic fit or tear exchange, so the small difference in clearance between groups, while statistically significant, may not have been the only factor correlated with midday fogging. A study by McKinney which enrolled 15 scleral lens wearers reported that the average corneal vault for patients with fogging was greater than those without fogging. ${ }^{3}$ When assessing the role of scleral lens fit in midday fogging, it is important to note that many studies may assess measurements, such as central clearance, with different procedures or definitions. For example, some studies measure clearance of the lens by estimation with biomicroscopy, while others may use anterior OCT or other technology to assist in the measurement. Even with the use of an objective imaging device such as OCT for these measurements, the definition of central clearance, or post-lens fluid reservoir, is often ambiguous. For example, it is unclear in some cases whether central clearance is defined as the amount of vault over the center of the lens, or the amount of vault over the decentered cone. These differences, and other variables left uncontrolled, such as haptic alignment, make it difficult to determine the effect of the thickness of the post-lens reservoir on midday fogging.

\section{Role of Tightness of Lenses}

The tightness of the scleral lens may play a role in midday fogging. Tightness of a scleral lens may be the result of the force of a scleral lens system, when, for example, a lens with a steep haptic burrows into the conjunctiva. The hypothesis of tightness playing a role in midday fogging can be further supported when evaluating the role of fenestration in scleral lenses fits. Fenestration of a lens was originally utilized in scleral lenses made of PMMA, and were essentially holes added to the lens in order to create air exchange and increased oxygen transmission to the post-lens tear reservoir. ${ }^{32}$ The fenestrations also served as a way to decrease suction of the lens to the ocular surface. A paper on the history of scleral lens fenestration focused on many years of scleral lens fitting by a single practitioner, and reported that fenestration eliminated debris collection under a lens. The paper noted that when debris did collect it was assumed to be a result of poorly designed or blocked fenestrations, which may imply that fenestrations could have allowed some tear exchange in addition to air exchange, and which suggests that fenestrations were unable to alleviate all cases of midday fogging. ${ }^{33}$ Despite the possible benefits of their use, fenestrations allow for the introduction of air bubbles under the lens that could block the visual axis, and may result in corneal desiccation. While necessary to increase oxygen transmission in early scleral lenses made with PMMA material, fenestration is not generally required today to allow for oxygen to reach the cornea, and the use of gaspermeable materials for scleral lenses has largely fulfilled the need to improve oxygen transmission. ${ }^{34}$ However, the concept that fenestrations reduce the pressure of the lens upon the eye and may also decrease midday fogging points to the possibility that inflammation due to a tight fit may be implicated in this phenomenon.

McKinney et al assessed the tightness of a lens fit and measured tear exchange in scleral lens wearers when comparing participants who had to remove lenses before 8 hours of lens wear each day due to presumed fogging to those who were able to wear their lenses for 8 or more continuous hours each day. The study found that $80 \%$ of the participants with fogging had tightly fitting lenses, compared to $40 \%$ of the wearers without interrupted wear. Interestingly, the tear exchange rate measured by fluorophotometry was the same in the two groups. ${ }^{3}$

\section{Tear Exchange}

Case reports and studies show conflicting results on the effect of tear exchange upon midday fogging. ${ }^{3,25,26,31}$ Multiple reports have given evidence that midday fogging was reduced or eliminated by fitting a lens more closely to the scleral contour and thereby, minimizing tear exchange..$^{25,26,35}$ It has been shown that the toricity of the sclera can be much greater than that of the cornea. ${ }^{36,37}$ Lenses with spherical haptic zones may have greater edge lift in different portions of the lens, resulting in "inadvertent" tear exchange that could "pump" tear debris under the lens. ${ }^{38}$ Additionally, the sclera may have a contour that is not symmetrical. To aid practitioners in fitting a lens to match the scleral contour, many recent lens designs allow for toric and quadrant specific haptic zones, and technology is available to create custom lenses based upon scleral topography ${ }^{38-40}$ or impressions of the eye. ${ }^{41}$ It is believed that fitting the haptic of the lenses to the scleral contour will minimize excessive tear exchange or "rocking" of lenses over mismatched scleral terrain. Despite these reports, two studies have found no differences when comparing tear exchange in groups of participants with and without midday fogging. ${ }^{3,31}$ Additionally, 
a study by the SCOPE group, which asked practitioners to report on their most recent scleral lens fit $(n=248)$, found that midday fogging occurred at the same rate in scleral lenses with spherical haptics compared to those with nonspherical haptic zones, including customized lenses and those made using impression-based fitting. ${ }^{42}$ Because lenses with non-spherical haptics are generally fit In order to match the contour, and thus reduce tear exchange, the results of this large survey are in line with the results of the clinical studies that also found no difference tear exchange when comparing participants with and without midday fogging. The conflict between the evidence shown in specific reports and the experimental findings may be due to the multiple factors and types of particulate that may contribute to midday fogging. It can be hypothesized that debris present in the tear film, such as lipid or mucin, may contribute to midday fogging in an open system with tear exchange, while epithelial cells or inflammatory cells may be trapped in the post-lens reservoir in patients who experience midday fogging and have no tear exchange. Studies of tear exchange coupled with identification of the debris could help to determine if different strategies are needed to mitigate different types of midday fogging.

\section{Supplemental Solution in the Post Lens Reservoir}

Some clinicians have reported success in decreasing midday fogging by adding a preservative-free high viscosity artificial tear to the saline instilled in the reservoir of the lens before insertion. ${ }^{43}$ While no formal studies have been conducted on this topic, clinician recommendation that "thick" artificial tears be added to the non-preserved filling solution before lens insertion is common. ${ }^{44,45}$ Various hypotheses have been given for why addition of this thick solution could decrease midday fogging, including possible changes in "nutritious ion presence," osmolality, or the difference in viscosity. ${ }^{45}$ In a study of tear reservoir turbidity, Carracedo et al raised the possibility that a more viscous solution could have an effect on conjunctival compression by acting as a cushion. ${ }^{11}$ A study by Courey compared the change in lens clearance over the cornea after insertion of a scleral lens filled with non-preserved methoxycellulose, compared to the clearance of a lens filled with non-preserved saline in the contralateral eye. ${ }^{46}$ The mean decrease in clearance over time was not significantly different when comparing the two types of filling solution. The study concluded that viscous eye drops do not affect settling of a scleral lens. Further study of scleral lens wear with viscous filling solution added to filling solution is needed to determine if midday fogging is changed.

\section{Inflammation as a Contributor to Midday Fogging}

In analyzing the known studies of midday fogging, it appears that inflammation plays a common role with many of the causative factors. This is consistent with the SCOPE group's study of midday fogging, in which redness or irritation with scleral lens wear was the only factor which was significant when comparing foggers to nonfoggers. ${ }^{42}$ Inflammation can lead to an increase in all hypothesized types of midday fogging particulate. It is possible that the presence of different types of particulate matter indicates different mechanisms of inflammation which may contribute to midday fogging, including solution incompatibility with corneal cells, friction or compression from excessive lens movement or rocking, or overall tightness and pressure put upon the ocular surface. The variation in the types of particulate may explain why there is not one simple way to treat midday fogging.

\section{Discussion}

Clearly, there are multiple causes of midday fogging and many large studies which assess multiple aspects of lens fit, solution use, tightness of lenses and assessments of inflammation are needed to definitively understand midday fogging. In the absence of that, some observations about the studies which have been conducted may help in formulating educated hypotheses on how to minimize midday fogging.

Some level of inflammation may be unavoidable in scleral lens-wearing patients, especially those with keratoconus and ocular surface disease. Because studies have shown that exposure to preservatives, vehicles for ophthalmic medications, and even tear substitutes which have ionic compositions different from tears can cause epithelial cell changes and cellular sloughing, ${ }^{13,47,48}$ it is reasonable to surmise that minimizing insults to the ocular system whether related to ocular health or scleral lens wear could aid in decreasing cellular debris in the post lens tear reservoir of scleral lens wearers. Treatment of ocular allergies, giant papillary conjunctivitis and meibomian gland dysfunction should be a priority for scleral lens wearers in order to decrease inflammation on the ocular surface. 
Additional factors that can contribute to inflammation may be related to improper solution use by the patient. It is important to regularly confirm that patients are using nonpreserved solutions when filling the reservoir of the lens before insertion. Often, price and convenience make other preserved saline or multipurpose contact lens solutions attractive, so it is important not to assume the patient is using the solution they were instructed to use when filling the lens. Those patients using non-preserved sodium chloride saline and experiencing midday fogging may benefit from switching to a $\mathrm{pH}$ buffered filling solution that matches the tear composition, as it minimizes epithelial cell sloughing compared to sodium chloride filling solution. Solution alone may reduce midday fogging, but it will not eliminate it in all patients.

Based upon Courey's research on lens settling with viscous solution in the post-lens reservoir, it does not appear that a viscous solution prevents the "suction" of a scleral lens due to settling. ${ }^{46}$ However, it is possible that a viscous solution could decrease friction in the case of a lens which impinges differentially at a position or positions around its circumference. This could result in less inflammation, which in turn could lessen cellular turnover. Future studies could examine the effect of adding a non-preserved viscous solution to the lens reservoir before insertion on inflammation and midday fogging for those patients in which excessive lens edge and conjunctival interaction occur.

Studies which have assessed the role of lens clearance on midday fogging have conflicting results. ${ }^{11,18,20,31}$ Studies which found a relationship between midday fogging and clearance did not control for other lens fitting parameters. Additionally, the lack of defined measurement methodologies of central clearance may add ambiguity to the results when measuring very small differences. Based upon theoretical models of oxygen transmissibility through tears and empirical studies of corneal edema, it is possible that excessive clearance in the absence of tear exchange could result in hypoxia, and therefore inflammation of the cornea. Similarly, studies have found increased inflammatory markers with excessive limbal clearance. ${ }^{18}$ Much more work needs to be done to understand the role of lens clearance upon midday fogging, particularly when controlling lens fit parameters such as tear exchange and tightness of fit.

While case reports have shown examples of decreases in midday fogging with toric or customized haptics designed to match the scleral contour, the rate of fogging remains the same with spherical and non-spherical haptic lens fits. ${ }^{42}$ The presence of eye redness was the only factor which corresponded to midday fogging. Therefore, if lens fit plays a role in midday fogging, it may be due to a lens that causes inflammation by different mechanisms. In the cases in which midday fogging is present and a lens is resting upon the ocular surface in one meridian, or a singular quadrant, it is possible that it may rock or "pump" tears under the lens edge during a blink with the "touch" points acting as a fulcrum or pressure point. Attempts to make these lenses fit more uniformly around the sclera, whether by adding toricity or by more closely custom fitting the haptic portion of the eye may lessen the lens movement that could be causing inflammation. This may also be the mechanism by which yellow or brown lipid or white fluffy tear debris could enter the post lens tear reservoir, as this particulate may be more likely to originate in the tear film, rather than from the cornea. In the cases of wearers of spherical lenses that are fit tightly, inflammation could contribute to midday fogging due to compression. It should be noted, however, that lenses that fit the scleral contour well might also sink into the conjunctiva at a greater rate, causing overall compression, which can be another source of inflammation. In these situations, evaluating the tightness of the lens fit is crucial. Because a tightly fit lens has a "sealed system," the debris in the post lens tear reservoir is more likely to be fine particulate resulting from sloughed epithelial cells and inflammatory cells, and less likely to have trapped mucin or lipid particulate originating from the tear film. Factors such as the compressibility of the conjunctiva over the sclera may play a role in whether a lens fits tightly, regardless of the attempts to match the scleral contour with a lens haptic. Observation of the lens fit as well as the appearance of the ocular surface after lens removal is important, and communication with patients about their experiences while wearing the lenses is critical to success, particularly when attempting to determine if a lens fit could be causing inflammation. For example, a patient who appears to have a well-fit lens after 1-2 hours of wear, but has difficultly removing a lens after longer hours of wear may have a lens that settles more than expected, resulting in a suction type of force upon the eye. These patients often complain of rebound redness after removing the lenses or remark upon their ability to easily see the compression ring on the conjunctiva after lens removal. Examining the relationship between the edge of the lens and the conjunctiva using anterior OCT can help to confirm if a lens is fitting too tightly. If a lens is fitting too tightly, flattening the haptic or utilizing a larger diameter lens may help to prevent the edge of the lens from sinking and creating a constant force on the ocular surface. 
In clinical practice, assessment of tear exchange in all scleral lens wearers with midday fogging can be helpful in fully examining the lens fit. Assessing tear exchange can help to identify if the particulate is likely derived from the tear film and flows into the post-lens reservoir, or if the debris is present in a sealed environment. This is best accomplished at a follow-up visit, in which the lens has been worn for several hours. Sodium fluorescein or lissamine green applied to the front surface of a fully settled lens and immediately observed by slit lamp can aid the practitioner in determining if tear uptake is occurring freely or minimally. For example, if no dye is present in the post-lens reservoir after five minutes, it is likely that the lens if fitting very tightly. In this situation, a patient with midday fogging may first benefit from utilizing a tear filling solution mimicking filling solution. If midday fogging persists, it may be appropriate to refit the lens in a way to promote even tear exchange, whether by flattening the haptic, adjusting the diameter, or utilizing a lens design with tear exchange promoting channels. Alternatively, dye that passes quickly behind the lens may indicate some lens movement or lift that was less apparent without the dye, and the presence of midday fogging in this situation could be mitigated by fitting the lens to better match the contour in all areas. Watching the dye movement can help determine if tear exchange is occurring at one portion of the haptic or if the entire haptic portion is fitting equally loosely. Table 1 contains descriptions of particulate and strategies for minimizing the symptoms of midday fogging in various scenarios.

Table I Particulate Found in Midday Fogging with Possible Sources and Strategies for Minimizing the Accumulation of Types of Particulate in Various Scenarios of Midday Fogging

\begin{tabular}{|c|c|c|}
\hline $\begin{array}{l}\text { Particulate } \\
\text { Appearance in Post } \\
\text { Lens Reservoir }\end{array}$ & Possible Source & Possible Steps Toward Mitigation \\
\hline $\begin{array}{l}\text { Fine, white diffuse } \\
\text { particulate }\end{array}$ & Sloughed corneal epithelial cells, inflammatory cells & $\begin{array}{l}\text { - Utilize a filling solution with the ionic composition of tears } \\
\text { - In the absence of tear exchange - Decrease tightness of lens } \\
\text { fit by increasing edge lift of the haptic or increasing dia- } \\
\text { meter or utilizing tear exchange channels in scleral lens } \\
\text { design } \\
\text { - In the presence of tear exchange - fit haptic to match } \\
\text { scleral contour } \\
\text { - Decrease clearance if excessive }\end{array}$ \\
\hline \multirow[t]{2}{*}{$\begin{array}{l}\text { Yellow or brown } \\
\text { droplets }\end{array}$} & $\begin{array}{l}\text { In the presence of tear exchange - excess meibum in } \\
\text { tear film }\end{array}$ & $\begin{array}{l}\text { - Assess eyelids and lashes for meibomian dysfunction and/or } \\
\text { blepharitis and treat appropriately } \\
\text { - Examine lens } 360^{\circ} \text { for areas of lens compression or impin- } \\
\text { gement: fit lenses to match the scleral contour without } \\
\text { excessive tightness } \\
\text { - Consider toric, quadrant specific, or custom peripheries if } \\
\text { edge lift is inconsistent } \\
\text { - Utilize a filling solution with the ionic composition of tears }\end{array}$ \\
\hline & $\begin{array}{l}\text { In the absence of tear exchange - possible excessive } \\
\text { lipid resulting from a disruption in lipid metabolism } \\
\text { related to inflammation }\end{array}$ & $\begin{array}{l}\text { - Reduce the tightness of lens fit by flattening the haptic } \\
\text { - Portion to increase edge lift or increase the diameter } \\
\text { - Utilize a filling solution with the ionic composition of tears } \\
\text { - Decrease central clearance if excessive }\end{array}$ \\
\hline Large white globules & $\begin{array}{l}\text { Hypothesized to be mucin, but no mucin was found in } \\
\text { a small laboratory of } 3 \text { turbid samples. } \\
\text { Present often with excessive tear exchange }\end{array}$ & $\begin{array}{l}\text { - Examine lens } 360^{\circ} \text { for areas of lens compression or impin- } \\
\text { gement: fit lenses to match the scleral contour without } \\
\text { excessive tightness } \\
\text { - Consider toric, quadrant specific, or custom peripheries if } \\
\text { edge lift is inconsistent } \\
\text { - Utilize a filling solution with the ionic composition of tears } \\
\text { - Decrease central clearance if excessive }\end{array}$ \\
\hline
\end{tabular}


Utilizing technology can aid in determining that a lens is not fitting too tightly or has variable areas of impingement or pressure on the eye. Anterior OCT can be used to selectively observe the edge of the lens in various positions around the eye to assess interactions with the conjunctiva. Scleral topography or tomography can assist in mapping the toricity of the sclera or can aid in lens design. ${ }^{38,39}$ It should be noted, however, that custom fitted lenses, even when created using an impression of the eye, can result in midday fogging. It is important to make sure that both the practitioner and patient have realistic goals when trying to refine the fit of these lenses.

\section{Conclusion}

Midday fogging with scleral lens wear is a common problem that requires careful examination of the ocular surface and lens fit, including assessments of tightness and tear exchange. Multiple approaches to mitigate inflammation may help to minimize the symptoms that accompany particulate accumulation in the post-lens reservoir. Identifying and treating sources of inflammation on the ocular surface and eyelids may lessen the collection of particulate under the lenses. Utilizing a filling solution that minimizes corneal epithelial cell inflammation and sloughing by matching the ionic composition of tears is a valuable tool in reducing the symptoms of midday fogging. Fitting of the haptic portion of the lens may play a role in midday fogging, but not in a "one-size-fits-all" mentality. While a scleral lens that matches the scleral contour yet does not compress tightly is the least likely to induce inflammation, this alone may not eliminate all sources of particulate collecting in the post-lens reservoir. Due to the complex ocular conditions of many scleral lens wearers, complete elimination of inflammation may not be possible, but minimization of inflammation and the resulting particulate can help to extend uninterrupted wear time in those patients who truly benefit from scleral lens wear.

\section{Disclosure}

No funding was received for the authorship of this paper. One study mentioned in this paper was completed by the author with funding from Contamac, Inc., although the funder had no role in the study design, analysis and interpretation of the cited paper, nor the current paper. The author has completed consulting work for Contamac and Alcon, reports grants and personal fees from Contamac,
Inc., outside the submitted work, and reports no other potential conflicts of interest for this work.

\section{References}

1. Schornack M, Helland M, Nau CB. Change in fluid reservoir turbidity at 20-minute intervals over 2 hours of small diameter scleral lens wear. Invest Ophth Vis Sci. 2015;56:6071.

2. Fogt JS, Karres M, Barr JT. Changes in symptoms of midday fogging with a novel scleral contact lens filling solution. Optom Vis Sci. 2020;97(9):690-696. doi:10.1097/OPX.0000000000001559

3. McKinney A, Miller W, Leach N, et al. The cause of midday visual fogging in scleral gas permeable lens wearers. Invest Ophthalmol Vis Sci. 2013;54:5483.

4. Rathi VM, Mandathara PS, Vaddavalli PK, et al. Fluid Filled scleral contact lens in pediatric patients: challenges and outcome. Cont Lens Anterior Eye. 2012;35(4):189-192. doi:10.1016/j.clae.2012.03.001

5. Schornack MM, Fogt J, Harthan J, et al. Factors Associated with Patient-Reported Midday Fogging in Established Scleral Lens Wearers. Cont Lens Anterior Eye. 2020;43(6):602-608. doi:10.1016/j.clae.2020.03.005

6. Carrasquillo KG, Lipson MJ, Ezekiel DJ, Johns LK. Scleral Lens Complications and Problem Solving. In: Barnett M, Johns LK, editors. Ophthalmology: Current and Future Developments. Bentham Science Publishers; 2017:303-345.

7. Efron N, Jones L, Bron AJ, et al. The Tfos International Workshop on Contact Lens Discomfort: report of the Contact Lens Interactions with the Ocular Surface and Adnexa Subcommittee. Invest Ophthalmol Vis Sci. 2013;54(11):TFOS98-TFOS122. doi:10.1167/ iovs.13-13187

8. Robertson DM, Cavanagh HD. The clinical and cellular basis of contact lens-related corneal infections: a review. Clin Ophthalmol. 2008;2:907-917. doi:10.2147/OPTH.S3249

9. Tse V, Tan B, Kim YH, et al. Tear dynamics under scleral lenses. Cont Lens Anterior Eye. 2019;42(1):43-48. doi:10.1016/j. clae.2018.11.016

10. Paugh JR, Chen E, Heinrich C, et al. Silicone hydrogel and rigid gas-permeable scleral lens tear exchange. Eye Contact Lens. 2018;44 (2):97-101. doi:10.1097/ICL.0000000000000400

11. Carracedo G, Serramito-Blanco M, Martin-Gil A, et al. Post-lens tear turbidity and visual quality after scleral lens wear. Clin Exp Optom. 2017;100(6):577-582. doi:10.1111/cxo.12512

12. Yu F, Liu X, Zhong Y, et al. Sodium hyaluronate decreases ocular surface toxicity induced by benzalkonium chloride-preserved latanoprost: an in vivo study. Invest Ophthalmol Vis Sci. 2013;54:3385-3393. doi:10.1167/iovs.12-11181

13. Bachman WG, Wilson G. Essential Ions for Maintenance of the Corneal Epithelial Surface. Invest Ophthalmol Vis Sci. 1985;26:1484-1488.

14. Paugh JR, Chen E, Heinrich C; Rismondo V, Osgood TB, Leering P, et al. Electrolyte composition of lacrimal gland fluid and tears of normal and vitamin a-deficient rabbits. CLAO J. 1989;15(3):222-228.

15. Fullard RJ, Wilson GS. Investigation of sloughed corneal epithelial-cells collected by noninvasive irrigation of the corneal surface. Curr Eye Res. 1986;5:847-856. doi:10.3109/02713688609029236

16. Montani G. Effects of different post lens saline filling solutions on midday fogging in habitual scleral lens wearers. Contact Lens Anterio. 2020;44:10. doi:10.1016/j.clae.2020.12.035

17. Li DQ, Lokeshwar BL, Solomon A, et al. Regulation of Mmp-9 Production by Human Corneal Epithelial Cells. Exp Eye Res. 2001;73:449-459. doi:10.1006/exer.2001.1054

18. Yeung D, Murphy PJ, Sorbara L. Comparative analysis of tear proteins in keratoconic scleral lens wearers with variation in limbal clearance. Optom Vis Sci. 2021;98(2):143-149. doi:10.1097/ OPX.0000000000001645 
19. Walker MK, Lema C, Redfern R. Scleral lens wear: measuring inflammation in the fluid reservoir. Cont Lens Anterior Eye. 2020;43:577-584. doi:10.1016/j.clae.2020.02.017

20. Postnikoff CK, Pucker AD, Laurent J, et al. Identification of leukocytes associated with midday fogging in the post-lens tear film of scleral contact lens wearers. Invest Ophthalmol Vis Sci. 2019;60 (1):226-233. doi:10.1167/iovs.18-24664

21. Galvis V, Sherwin T, Tello A, et al. Keratoconus: an Inflammatory Disorder? Eye. 2015;29(7):843-859. doi:10.1038/eye.2015.63

22. Walker M. Scleral Lenses, Clearing the Fog. ISITE Online J. 2014.

23. Willcox MDP. Tear Film, Contact Lenses and Tear Biomarkers. Clinical and Experimental Optometry. 2019;102(4):350-363. doi:10.1111/cxo.12918

24. van Diepen JA, Berbee JFP, Havekes LM, Rensen PCN. Interactions between Inflammation and Lipid Metabolism: relevance for Efficacy of Anti-Inflammatory Drugs in the Treatment of Atherosclerosis. Atherosclerosis. 2013;228:306-315. doi:10.1016/j.atherosclerosis. 2013.02.028

25. Sonsino J, Reinoso G, Teller R. Proposed Method to Eliminate Debris in the Scleral Post-Lens Tear Reservoir: Case Report. In: Global Specialty Lens Symposium. Las Vegas, NV: Las Vegas, NV; 2018.

26. Sonsino J, Sclafani LA. Stopping a Scleral Lens from Rockin' and Rollin' on a Highly Toric Sclera. In: Global Specialty Lens Symposium. Las Vegas, NV: Las Vegas, NV; 2019.

27. Michaud L, van der Worp E, Brazeau D, et al. Predicting Estimates of Oxygen Transmissibility for Scleral Lenses. Cont Lens Anterior Eye. 2012;35(6):266-271. doi:10.1016/j.clae.2012.07.004

28. Fisher D, Collins MJ, Vincent SJ. Fluid reservoir thickness and corneal oedema during closed eye scleral lens wear: experimental and theoretical outcomes. Cont Lens Anterior Eye. 2021;44 (1):124-125. doi:10.1016/j.clae.2020.10.004

29. Fisher D, Collins MJ, Vincent SJ. Fluid reservoir thickness and corneal edema during open-eye scleral lens wear. Optom Vis Sci. 2020;97(9):683-689. doi:10.1097/OPX.0000000000001558

30. Vincent SJ, Alonso-Caneiro D, Collins MJ. The time course and nature of corneal oedema during sealed miniscleral contact lens wear. Cont Lens Anterior Eye. 2019;42(1):49-54. doi:10.1016/j. clae.2018.03.001

31. Skidmore KV, Walker MK, Marsack JD, et al. A measure of tear inflow in habitual scleral lens wearers with and without midday fogging. Contact Lens Anterio. 2019;42:36-42. doi:10.1016/j. clae.2018.10.009

32. Ezekiel D. Gas Permeable Haptic Lenses. Journal of the British Contact Lens Association. 1983;6:158. doi:10.1016/S0141-7037(83)80064-0

33. Fadel D, Ezekiel DF. Fenestrated scleral lenses: back to the origins? review of their benefits and fitting techniques. Optom Vis Sci. 2020;97(9):807-820. doi:10.1097/OPX.0000000000001562

34. Rosenthal P, Croteau A. Fluid-ventilated, gas-permeable scleral contact lens is an effective option for managing severe ocular surface disease and many corneal disorders that would otherwise require penetrating keratoplasty. Eye Contact Lens. 2005;31(3):130-134. doi:10.1097/01.ICL.0000152492.98553.8D

Clinical Optometry

\section{Publish your work in this journal}

Clinical Optometry is an international, peer-reviewed, open access journal publishing original research, basic science, clinical and epidemiological studies, reviews and evaluations on clinical optometry. All aspects of patient care are addressed within the journal as well as the practice of optometry including economic and business analyses. Basic and clinical research papers are published that cover

Submit your manuscript here: https://www.dovepress.com/clinical-optometry-journal
35. Conway M. An Update on Scleral Lenses. In. Global Insight. Curr Opinion Ophthalmol. 2008;19(4):298.

36. DeNaeyer G, Sanders DR, van der Worp E, et al. Qualitative assessment of scleral shape patterns using a new wide field ocular surface elevation topographer: the sssg study. J Cont Lens Res Sci. 2017;1:12-22. doi:10.22374/jclrs.v1i1.11

37. Ritzmann M, Caroline PJ, Borret R, Korszen E. An analysis of anterior scleral shape and its role in the design and fitting of scleral contact lenses. Cont Lens Anterior Eye. 2018;41(2):205-213. doi:10.1016/j.clae.2017.10.010

38. DeNaeyer G, Sanders DR, Farajian TS. Surface coverage with single vs. multiple gaze surface topography to fit scleral lenses. Cont Lens Anterior Eye. 2017;40(3):162-169. doi:10.1016/j.clae.2017.03.009

39. Jesus DA, Kedzia R, Iskander DR. Precise measurement of scleral radius using anterior eye profilometry. Cont Lens Anterior Eye. 2017;40(1):47-52. doi:10.1016/j.clae.2016.11.003

40. Bandlitz S, Esper P, Stein M, et al. Corneoscleral Topography Measured with Fourier-Based Profilometry and Scheimpflug Imaging. Optom Vis Sci. 2020;97(9):766-774. doi:10.1097/OPX.00 00000000001572

41. Nau A, Shorter ES, Harthan JS. et al. Multicenter Review of Impression-Based Scleral Devices. Cont Lens Anterior Eye;2020. 101380. doi:10.1016/j.clae.2020.10.010

42. Schornack MM, Fogt J, Harthan J, et al. Factors associated with patient-reported midday fogging in established scleral lens wearers. Cont Lens Anterior Eye. 2020;43(6):602-608.

43. Fadel D. Scleral Lens Issues and Complications Related to a Non-Optimal Fitting Relationship between the Lens and Ocular Surface. Eye \& Contact Lens-Science and Clinical Practice. 2019;45:152-163. doi:10.1097/ICL.0000000000000523

44. Nichols JJ. Education highlights from the 2015 gsls specialty contact lens education reigned supreme at this year's record-breaking conference. Contact Lens Spectrum. 2015;30:22-30.

45. Walker MK, Bergmanson JP, Miller WL, et al. Complications and fitting challenges associated with scleral contact lenses: a review. Cont Lens Anterior Eye. 2016;39(2):88-96. doi:10.1016/j.clae.20 15.08 .003

46. Courey C, Michaud L. Variation of clearance considering viscosity of the solution used in the reservoir and following scleral lens wear over time. Cont Lens Anterior Eye. 2017;40:260-266. doi:10.1016/j. clae.2017.03.003

47. Pfister RR, Burstein N. The effects of ophthalmic drugs, vehicles, and preservatives on corneal epithelium: a scanning electron microscope study. Invest Ophthalmol. 1976;15:246-259.

48. Dormans JA, van Logten MJ. The effects of ophthalmic preservatives on corneal epithelium of the rabbit: a scanning electron microscopical study. Toxicol Appl Pharmacol. 1982;62:251-261. doi:10.1016/0041008X(82)90123-5 all aspects of optics, refraction and its application to the theory and practice of optometry. The manuscript management system is completely online and includes a very quick and fair peer-review system, which is all easy to use. Visit http://www.dovepress.com/ testimonials.php to read real quotes from published authors. 